KEK-TH-412

September 1994

\title{
Status of Higher-Order Corrections in the Standard Electroweak Theory*
}

\author{
Bernd A. Kniehl ${ }^{\dagger \ddagger}$ \\ Theory Division, National Laboratory for High Energy Physics (KEK), \\ 1-1 Oho, Tsukuba-shi, Ibaraki-ken, 305 Japan
}

\begin{abstract}
We review recent theoretical progress in the computation of radiative corrections beyond one loop within the standard model of electroweak interactions, both in the gauge and Higgs sectors. In the gauge sector, we discuss universal corrections of $\mathcal{O}\left(G_{F}^{2} M_{H}^{2} M_{W}^{2}\right), \mathcal{O}\left(G_{F}^{2} m_{t}^{4}\right), \mathcal{O}\left(\alpha_{s} G_{F} M_{W}^{2}\right), \mathcal{O}\left(\alpha_{s}^{2} G_{F} m_{t}^{2}\right)$, and those due to virtual $t \bar{t}$ threshold effects, as well as specific corrections to $\Gamma(Z \rightarrow b \bar{b})$ of $\mathcal{O}\left(G_{F}^{2} m_{t}^{4}\right), \mathcal{O}\left(\alpha_{s} G_{F}\right.$ $\left.m_{t}^{2}\right)$, and $\mathcal{O}\left(\alpha_{s}^{3}\right)$ including finite- $m_{b}$ effects. We also present an update of the hadronic contributions to $\Delta \alpha$. Theoretical uncertainties, other than those due to the lack of knowledge of $M_{H}$ and $m_{t}$, are estimated. In the Higgs sector, we report on the $\mathcal{O}\left(\alpha_{s} G_{F} m_{t}^{2}\right)$ corrections to $\Gamma(H \rightarrow f \bar{f})$ including those which are specific for the $b \bar{b}$ mode, the $\mathcal{O}\left(\alpha_{s}^{2}\right)$ corrections to $\Gamma(H \rightarrow q \bar{q})$ including the finite- $m_{q}$ terms, and the $\mathcal{O}\left(\alpha_{s}\right)$ corrections to $\Gamma(H \rightarrow g g)$.
\end{abstract}

\section{Introduction}

Precision tests of the standard electroweak theory [1] have reached a mature stage since their beginnings more than two decades ago. This topic is routinely on the agenda of the major high-energy-physics conferences and workshops [2], and numerous comprehensiveand in some cases even encyclopaedic - reviews on the status of quantum corrections have been published in the recent few years [3, 4, 3, 6, 7, 8, 8]. In this article, an attempt is made to summarize the latest developments in the study of higher-order corrections to the electroweak parameters and the partial decay widths of the $Z$ and Higgs bosons in the framework of the minimal standard model. In the case of the Higgs boson, we shall concentrate on the low- and intermediate mass range, $M_{H}<2 M_{W}$, which will be accessed by colliding-beam experiments in the near future.

*To appear in Int. J. Mod. Phys. A.

${ }^{\dagger}$ On leave from II. Institut für Theoretische Physik, Universität Hamburg, Luruper Chaussee 149, 22761 Hamburg, Germany; address after 1 October 1994: Max-Planck-Institut für Physik, Föhringer Ring 6, 80805 Munich, Germany.

${ }^{\ddagger}$ JSPS Fellow. 
As a rule, the size of radiative corrections to a given process is determined by the discrepancy between the various mass and energy scales involved. In $Z$-boson physics, the dominant effects arise from light charged fermions, which induce large logarithms of the form $\alpha^{n} \ln ^{m}\left(M_{Z}^{2} / m_{f}^{2}\right)(m \leq n)$ in the fine-structure constant (and also in initial-state radiative corrections), and from the top quark, which generates power corrections of the orders $G_{F} m_{t}^{2}, G_{F}^{2} m_{t}^{4}, \alpha_{s} G_{F} m_{t}^{2}$, etc. On the other hand, the quantum effects due to a heavy Higgs boson are screened, i.e., logarithmic in $M_{H}$ at one loop and just quadratic at two loops. By contrast, such corrections are proportional to $M_{H}^{2}$ and $M_{H}^{4}$, respectively, in the Higgs sector.

\section{Gauge Sector}

\subsection{Universal Corrections: Electroweak Parameters (Oblique Corrections)}

For a wide class of low-energy and $Z$-boson observables, the dominant effects originate entirely in the gauge-boson propagators (oblique corrections) and may be parametrized conveniently in terms of four electroweak parameters, $\Delta \alpha, \Delta \rho, \Delta r$, and $\Delta \kappa$, which bear the following physical meanings [5]:

1. $\Delta \alpha$ determines the running fine-structure constant at the $Z$-boson scale, $\alpha\left(M_{Z}\right) / \alpha=$ $(1-\Delta \alpha)^{-1}$, where $\alpha$ is the corresponding value at the electron scale;

2. $\Delta \rho$ measures the quantum corrections to the ratio of the neutral- and charged-current amplitudes at low energy, $G_{N C}(0) / G_{C C}(0)=(1-\Delta \rho)^{-1}$ [9];

3. $\Delta r$ embodies the non-photonic corrections to the muon lifetime, $G_{F}=\left(\pi \alpha / \sqrt{2} s_{w}^{2}\right.$ $\left.M_{W}^{2}\right)(1-\Delta r)^{-1}[10$;

4. $\Delta \kappa$ controls the effective weak mixing angle, $\bar{s}_{w}^{2}=s_{w}^{2}(1+\Delta \kappa)$, that occurs in the ratio of the $f \bar{f} Z$ vector and axial-vector couplings, $v_{f} / a_{f}=1-4\left|Q_{f}\right| \bar{s}_{w}^{2}$ 四].

Unless stated otherwise, we adopt the on-shell scheme [11] and set $c_{w}^{2}=1-s_{w}^{2}=M_{W}^{2} / M_{Z}^{2}$ [10]. The large logarithms are collected by $\Delta \alpha$, and the leading $m_{t}$ dependence is carried by $\Delta \rho . \Delta r$ and $\Delta \kappa$ may be decomposed as $(1-\Delta r)=(1-\Delta \alpha)\left(1+c_{w}^{2} / s_{w}^{2} \Delta \rho\right)-\Delta r_{r e m}$ and $\Delta \kappa=c_{w}^{2} / s_{w}^{2} \Delta \rho+\Delta \kappa_{\text {rem }}$, respectively, where the remainder parts are devoid of $m_{f}$ logarithms and $m_{t}$ power terms. The triplet $\left(\Delta \rho, \Delta r_{w}, \Delta \kappa\right)$, where $\Delta r_{w}$ is defined by $(1-\Delta r)=$ $(1-\Delta \alpha)\left(1-\Delta r_{w}\right)$, is equivalent to synthetical sets like $(S, T, U)$ and $\left(\varepsilon_{1}, \varepsilon_{2}, \varepsilon_{3}\right)$ [12], which have gained vogue recently. We note in passing that the bosonic contributions to these electroweak parameters are, in general, gauge dependent and finite only in a restricted class of gauges if the conventional formulation in terms of vacuum polarizations is employed. This problem may be cured in the framework of the pinch technique [13].

At two loops, large contributions are expected to arise from the exchange of heavy Higgs bosons, heavy top quarks, and gluons. The hadronic contributions to $\Delta \alpha$ and the $t \bar{t}$ threshold effects on $\Delta \rho, \Delta r$, and $\Delta \kappa$ cannot be calculated reliably in QCD to finite order. However, 
they may be related via dispersion relations to data of $e^{+} e^{-} \rightarrow$ hadrons and theoretical predictions of $e^{+} e^{-} \rightarrow t \bar{t}$ based on realistic quark potentials, respectively.

\subsubsection{Two-Loop $\mathcal{O}\left(G_{F}^{2} M_{H}^{2} M_{Z}^{2}\right)$ Corrections}

Such corrections are generated by two-loop gauge-boson vacuum-polarization diagrams that are constructed from physical and unphysical Higgs bosons. Knowledge 14 of the first two terms of the Taylor expansion around $q^{2}=0$ is sufficient to derive the leading contributions to $\Delta \rho, \Delta r$, and $\Delta \kappa[7,15]$,

$$
\begin{aligned}
\Delta \rho & =\frac{G_{F}^{2} M_{H}^{2} M_{W}^{2}}{64 \pi^{4}} \frac{s_{w}^{2}}{c_{w}^{2}}\left[-9 \sqrt{3} \operatorname{Li}_{2}\left(\frac{\pi}{3}\right)+\frac{9}{2} \zeta(2)+\frac{9}{4} \pi \sqrt{3}-\frac{21}{8}\right] \\
& \approx 4.92 \cdot 10^{-5}\left(\frac{M_{H}}{1 \mathrm{TeV}}\right)^{2}, \\
\Delta r & =\frac{G_{F}^{2} M_{H}^{2} M_{W}^{2}}{64 \pi^{4}}\left[9 \sqrt{3} \mathrm{Li}_{2}\left(\frac{\pi}{3}\right)-\frac{25}{18} \zeta(2)-\frac{11}{4} \pi \sqrt{3}+\frac{49}{72}\right] \\
& \approx-1.05 \cdot 10^{-4}\left(\frac{M_{H}}{1 \mathrm{TeV}}\right)^{2}, \\
\Delta \kappa & =\frac{G_{F}^{2} M_{H}^{2} M_{W}^{2}}{64 \pi^{4}}\left[-9 \sqrt{3} \mathrm{Li}_{2}\left(\frac{\pi}{3}\right)+\frac{53}{18} \zeta(2)+\frac{5}{2} \pi \sqrt{3}-\frac{119}{72}\right] \\
& \approx 1.37 \cdot 10^{-4}\left(\frac{M_{H}}{1 \mathrm{TeV}}\right)^{2},
\end{aligned}
$$

Due to the smallness of the prefactors, these contributions are insignificant for $M_{H} \lesssim 1 \mathrm{TeV}$.

\subsubsection{Two-Loop $\mathcal{O}\left(G_{F}^{2} m_{t}^{4}\right)$ Corrections for $M_{H} \neq 0$}

Also at two loops, $\Delta \rho$ picks up the leading large- $m_{t}$ term, and $\Delta r$ and $\Delta \kappa$ depend on $m_{t}$ chiefly via $\Delta \rho$. Neglecting $m_{b}$ and defining $x_{t}=\left(G_{F} m_{t}^{2} / 8 \pi^{2} \sqrt{2}\right)$, one has

$$
\Delta \rho=3 x_{t}\left[1+x_{t} \rho^{(2)}\left(\frac{M_{H}}{m_{t}}\right)-\frac{2}{3}(2 \zeta(2)+1) \frac{\alpha_{s}\left(m_{t}\right)}{\pi}\right],
$$

where, for completeness, also the well-known $\mathcal{O}\left(\alpha_{s} G_{F} m_{t}^{2}\right)$ term [16, 17] is included. Very recently, also the $\mathcal{O}\left(\alpha_{s}^{2} G_{F} m_{t}^{2}\right)$ term has been computed [18], the result being $(-21.27063+$ $\left.1.78621 N_{F}\right)\left(\alpha_{s} / \pi\right)^{2}$, where $N_{F}$ is the number of active quark flavours. The contribution to this term due to the so-called double-triangle diagram was originally calculated in Ref. [19]. The coefficient $\rho^{(2)}(r)$ [20,21] is negative for all plausible values of $r$, bounded from below by $\rho^{(2)}(5.72)=-11.77$, and exhibits the following asymptotic behaviour:

$$
\rho^{(2)}(r)= \begin{cases}-12 \zeta(2)+19-4 \pi r+\mathcal{O}\left(r^{2} \ln r\right), & \text { if } r \ll 1 \\ 6 \ln ^{2} r-27 \ln r+6 \zeta(2)+\frac{49}{4}+O\left(\frac{\ln ^{2} r}{r^{2}}\right), & \text { if } r \gg 1\end{cases}
$$

The value at $r=0$ [22] greatly underestimates the effect. Both $\mathcal{O}\left(G_{F}^{2} m_{t}^{4}\right)$ and $\mathcal{O}\left(\alpha_{s} G_{F} m_{t}^{2}\right)$ corrections screen the one-loop result and thus increase the value of $m_{t}$ predicted indirectly 
from global analyses of low-energy, $M_{W}$, LEP/SLC, and other high-precision data. Recently, a first attempt was made to control subleading corrections to $\Delta \rho$, of $\mathcal{O}\left(G_{F}^{2} m_{t}^{2} M_{Z}^{2} \ln \left(M_{Z}^{2} / m_{t}^{2}\right)\right)$, in an SU(2) model of weak interactions, and significant effects were found [21].

\subsubsection{Two-Loop $\mathcal{O}\left(\alpha_{s} G_{F} M_{W}^{2}\right)$ Corrections}

For $m_{t} \gg M_{W}$, the bulk of the QCD corrections is concentrated in $\Delta \rho$ [see Eq. (2)]. However, for realistic values of $m_{t}$, the subleading terms, of $\mathcal{O}\left(\alpha_{s} G_{F} M_{W}^{2}\right)$, are significant numerically, e.g., they amount to $20 \%$ of the full two-loop QCD correction to $\Delta r$ at $m_{t}=$ $150 \mathrm{GeV}$. Specifically, one has [7,17]

$$
\begin{aligned}
& \Delta r_{r e m}=\frac{G_{F} M_{W}^{2}}{\pi^{3} \sqrt{2}}\left\{-\alpha_{s}\left(M_{Z}\right)\left(\frac{c_{w}^{2}}{s_{w}^{2}}-1\right) \ln c_{w}^{2}+\alpha_{s}\left(m_{t}\right)\left[\left(\frac{1}{3}-\frac{1}{4 s_{w}^{2}}\right) \ln \frac{m_{t}^{2}}{M_{Z}^{2}}+A+\frac{B}{s_{w}^{2}}\right]\right\} \\
& \Delta \kappa_{r e m}=\frac{G_{F} M_{W}^{2}}{\pi^{3} \sqrt{2}}\left\{\alpha_{s}\left(M_{Z}\right) \frac{c_{w}^{2}}{s_{w}^{2}} \ln c_{w}^{2}-\alpha_{s}\left(m_{t}\right)\left[\left(\frac{1}{6}-\frac{1}{4 s_{w}^{2}}\right) \ln \frac{m_{t}^{2}}{M_{Z}^{2}}+\frac{A}{2}+\frac{B}{s_{w}^{2}}\right]\right\}
\end{aligned}
$$

where terms of $\mathcal{O}\left(M_{Z}^{2} / m_{t}^{2}\right)$ are omitted within the square brackets and

$$
\begin{aligned}
A & =\frac{1}{3}\left[-4 \zeta(3)+\frac{4}{3} \zeta(2)+\frac{5}{2}\right] \approx-0.03833, \\
B & =\zeta(3)-\frac{2}{9} \zeta(2)-\frac{1}{4} \approx 0.58652 .
\end{aligned}
$$

For contributions due to the $t b$ doublet, $\mu=m_{t}$ is the natural renormalization scale for $\alpha_{s}(\mu)$.

\subsubsection{Hadronic Contributions to $\Delta \alpha$}

Jegerlehner has updated his 1990 analysis [6] of the hadronic contributions to $\Delta \alpha$ by taking into account the hadronic resonance parameters specified in the 1992 report [23] by the Particle Data Group and recently published low-energy $e^{+} e^{-}$data taken at Novosibirsk. The (preliminary) result at $\sqrt{s}=91.175 \mathrm{GeV}$ reads 24]

$$
\Delta \alpha_{\text {hadrons }}=0.0283 \pm 0.0007 \text {, }
$$

i.e., the central value has increased by $1 \cdot 10^{-4}$, while the error has decreased by $\pm 2 \cdot 10^{-4}$. The latter is particularly important, since this error has long constituted the dominant uncertainty for theoretical predictions of electroweak parameters. For comparison, we list the leptonic contribution up to two loops in QED [17],

$$
\begin{aligned}
\Delta \alpha_{\text {leptons }} & =\frac{\alpha}{3 \pi} \sum_{\ell}\left\{\ln \frac{M_{Z}^{2}}{m_{\ell}^{2}}-\frac{5}{3}+\frac{\alpha}{\pi}\left[\frac{3}{4} \ln \frac{M_{Z}^{2}}{m_{\ell}^{2}}+3 \zeta(2)-\frac{5}{8}\right]+O\left(\frac{m_{\ell}^{2}}{M_{Z}^{2}}\right)\right\} \\
& =0.0314966 \pm 0.0000004
\end{aligned}
$$

where the error stems from the current $m_{\tau}$ world average, $m_{\tau}=(1777.0 \pm 0.4) \mathrm{MeV}$ [25].

\subsection{5 tz Threshold Effects}

Although loop amplitudes involving the top quark are mathematically well behaved, it is evident that interesting and possibly significant features connected with the $t \bar{t}$ threshold 
cannot be accommodated when the perturbation series is truncated at finite order. In fact, perturbation theory up to $\mathcal{O}\left(\alpha \alpha_{s}\right)$ predicts a discontinuous steplike threshold behaviour for $\sigma\left(e^{+} e^{-} \rightarrow t \bar{t}\right)$. A more realistic description includes the formation of toponium resonances by multi-gluon exchange. For $m_{t} \gtrsim 130 \mathrm{GeV}$, the revolution period of a $t \bar{t}$ bound state exceeds its lifetime, and the individual resonances are smeared out to a coherent structure. By Cutkosky's rule [26], $\sigma\left(e^{+} e^{-} \rightarrow t \bar{t}\right)$ corresponds to the absorptive parts of the photon and $Z$-boson vacuum polarizations, and its enhancement at threshold induces additional contributions in the corresponding real parts, which can be computed via dispersive techniques. Decomposing the vacuum-polarization tensor generated by the insertion of a top-quark loop into a gauge-boson line as

$$
\Pi_{\mu \nu}^{V, A}(q)=\Pi^{V, A}\left(q^{2}\right) g_{\mu \nu}+\lambda^{V, A}\left(q^{2}\right) q_{\mu} q_{\nu}
$$

where $V$ and $A$ label the vector and axial-vector components and $q$ is the external fourmomentum, and imposing Ward identities, one derives the following set of dispersion relations [27,28]:

$$
\begin{aligned}
& \Pi^{V}\left(q^{2}\right)=\frac{q^{2}}{\pi} \int \frac{d s}{s} \frac{\operatorname{Im} \Pi^{V}(s)}{q^{2}-s-i \epsilon} \\
& \Pi^{A}\left(q^{2}\right)=\frac{1}{\pi} \int d s\left[\frac{\operatorname{Im} \Pi^{A}(s)}{q^{2}-s-i \epsilon}+\operatorname{Im} \lambda^{A}(s)\right] .
\end{aligned}
$$

The alternative set of dispersion relations proposed in Ref. [29] does not, in general, yield correct results, as has been demonstrated by establishing a perturbative counterexample, namely the $\mathcal{O}\left(\alpha_{s} G_{F} m_{t}^{2}\right)$ corrections to $\Gamma\left(H \rightarrow \ell^{+} \ell^{-}\right)$(see Sect. 3.1.1) [30]. In Ref. [31], it has been suggested that this argument may be extended to all orders in $\alpha_{s}$ by means of the operator product expansion. In the threshold region, only $\operatorname{Im} \Pi^{V}\left(q^{2}\right)$ and $\operatorname{Im} \lambda^{A}\left(q^{2}\right)$ receive significant contributions and are related by $\operatorname{Im} \lambda^{A}\left(q^{2}\right) \approx-\operatorname{Im} \Pi^{V}\left(q^{2}\right) / q^{2}$, while $\operatorname{Im} \Pi^{A}\left(q^{2}\right)$ is strongly suppressed due to centrifugal barrier effects [28]. Of course, $\lambda^{V}\left(q^{2}\right)=-\Pi^{V}\left(q^{2}\right) / q^{2}$ by the relevant Ward identity [27]. These contributions in turn lead to shifts in $\Delta \rho, \Delta r$, and $\Delta \kappa$. A crude estimation may be obtained by setting $\operatorname{Im} \Pi^{V}\left(q^{2}\right)=\operatorname{Im} \Pi^{V}\left(4 m_{t}^{2}\right)=\alpha_{s} m_{t}^{2}$ in the interval $\left(2 m_{t}-\Delta\right)^{2} \leq q^{2} \leq 4 m_{t}^{2}$, where $\Delta$ may be regarded as the binding energy of the $1 \mathrm{~S}$ state. This yields

$$
\begin{aligned}
& \Delta \rho=-\frac{G_{F}}{2 \sqrt{2}} \frac{\alpha_{s}}{\pi} m_{t} \Delta \\
& \Delta r=-\frac{c_{w}^{2}}{s_{w}^{2}} \Delta \rho\left[1-\left(1-\frac{8}{3} s_{w}^{2}\right)^{2} \frac{M_{Z}^{2}}{4 m_{t}^{2}-M_{Z}^{2}}+\frac{16}{9} s_{w}^{4} \frac{M_{Z}^{2}}{m_{t}^{2}}\right] \\
& \Delta \kappa=\frac{c_{w}^{2}}{s_{w}^{2}} \Delta \rho\left[1-\left(1-\frac{8}{3} s_{w}^{2}\right) \frac{M_{Z}^{2}}{4 m_{t}^{2}-M_{Z}^{2}}\right] .
\end{aligned}
$$

Obviously, the threshold effects have the same sign as the $\mathcal{O}\left(\alpha_{s} G_{F} m_{t}^{2}\right)$ corrections. For realistic quark potentials, one has approximately $\Delta \propto m_{t}$, so that the threshold contributions scale like $m_{t}^{2}$. Again, $\Delta \rho$ is most strongly affected, while the corrections to $\Delta r_{r e m}$ and 
$\Delta \kappa_{\text {rem }}$ are suppressed by $M_{Z}^{2} / m_{t}^{2}$. A comprehensive numerical analysis may be found in Refs. [28,32,33]. For $150 \mathrm{GeV} \leq m_{t} \leq 200 \mathrm{GeV}$, the threshold effects enhance the QCD corrections by roughly $30 \%$.

We emphasize that the above QCD corrections come with both experimental and theoretical errors. In the following, we shall estimate them for $\Delta \rho$ assuming $m_{t}=174 \mathrm{GeV}$ [34]; the results are similar for other universal electroweak parameters. The experimental errors are governed by the $\alpha_{s}$ measurement, $\alpha_{s}\left(M_{Z}\right)=0.118 \pm 0.006$ [35]. This amounts to errors of $\pm 5 \%$ and $\pm 18 \%$ on the continuum and threshold contributions to $\Delta \rho$, respectively. This reflects the fact the $\alpha_{s}$ dependence is linear in the continuum, while that of 1S peak height is approximately cubic. Since these errors are strongly correlated, we add them linearly to obtain an overall experimental error of $\pm 8 \%$ on the QCD correction to $\Delta \rho$. Theoretical errors are due to unknown higher-order corrections. In the continuum, they are usually estimated by varying the renormalization scale, $\mu$, of $\alpha_{s}(\mu)$ in the range $m_{t} / 2 \leq \mu \leq 2 m_{t}$, which amounts to $\pm 11 \%$. The theoretical error on the threshold contribution is mainly due to model dependence and is estimated to be $\pm 20 \%$ by comparing conventional quark potentials. For $m_{t}$ as large as $174 \mathrm{GeV}$, there is also a minor uncertainty connected with the choice of the lower bound of the dispersion integral, which, in Ref. [28], was chosen to be $s=\left(2 m_{t}-\Delta\right)^{2}$ with $\Delta=\sqrt{m_{t} \Gamma_{t}}$, which is $15.6 \mathrm{GeV}$ for $m_{t}=174 \mathrm{GeV}$. Variation of $\Delta$ in the wide range between $\sqrt{m_{t} \Gamma_{t}} / 2$ and $2 \sqrt{m_{t} \Gamma_{t}}$ induces a shift of $\pm 9 \%$ in the threshold contribution. Combining the common experimental error and the theoretical errors quadratically, we obtain a total error of $\pm 12 \%$ on the QCD correction to $\Delta \rho$. This corresponds to an absolute QCD-related uncertainty in $\Delta \rho$ of $\pm 1.5 \cdot 10^{-4}$. Due to the magnification factor $c_{w}^{2} / s_{w}^{2}$, the corresponding error on $\Delta r$ and $\Delta \kappa$ is $\pm 5.0 \cdot 10^{-4}$. We stress that, in the case of $\Delta r$ and thus the $M_{W}$ prediction from the muon lifetime, this error is almost as large as the one from hadronic sources introduced via $\Delta \alpha$ [see Eq. (6)]. For higher $m_{t}$ values, it may even be larger.

In Eq. (2), we have evaluated the $\mathcal{O}\left(\alpha_{s} G_{F} m_{t}^{2}\right)$ correction at $\mu=m_{t}$, since this is the only scale available. However, this is a leading-order QCD prediction, which suffers from the usual scale ambiguity. We may choose $\mu=\xi m_{t}$ in such a way that the $\mathcal{O}\left(\alpha_{s}(\mu) G_{F} m_{t}^{2}\right)$ calculation agrees with the $\mathcal{O}\left(\alpha_{s}\left(m_{t}\right) G_{F} m_{t}^{2}\right)$ one plus the $t \bar{t}$ threshold effects. In the case of $\Delta \rho$, this leads to $\xi=0.190_{-0.057}^{+0.097}$, where we have included a $\pm 30 \%$ error on the $t \bar{t}$ threshold contribution. Alternative, conceptually very different approaches of scale setting [36, 37,38] yield results in the same ball park. In Ref. [36], it is suggested that long-distance effects lower the renormalization point for $\alpha_{s}(\mu)$ in Eq. (2) through the contributions of the nearmass-shell region to the evolution of the quark mass from the mass shell to distances of order $1 / m_{t}$. To estimate these effects, the authors of Ref. [36] apply the Brodsky-LepageMackenzie (BLM) criterion [39] to Eq. (2) and find $\xi=0.154$. The author of Ref. [37] expresses first the fermionic contribution to $\Delta \rho$ [see Eq. (2)] in terms of $\bar{m}_{t}\left(m_{t}\right)$, where $\bar{m}_{t}(\mu)$ is the top-quark $\overline{\mathrm{MS}}$ mass at renormalization scale $\mu$, and then relates $\bar{m}_{t}\left(m_{t}\right)$ to $m_{t}$ by optimizing the expansion of $m_{t} / \bar{m}_{t}\left(m_{t}\right)$, which is known through $\mathcal{O}\left(\alpha_{s}^{2}\right)$ [see Eq. (23)] [40,41], according to the BLM criterion [39]. In Ref. [38], he refines this argument by using the new results of Ref. [18] and an expansion of $\mu_{t} / \bar{m}_{t}\left(m_{t}\right)$, where $\mu_{t}=\bar{m}_{t}\left(\mu_{t}\right)$, and obtains 
$\xi=0.323$. He argues that an important advantage of this approach is that the coefficients of $\left(\alpha_{s} / \pi\right)^{n}(n=1,2)$ in the final expressions are either very small or of $\mathcal{O}(1)$. In particular, when $\Delta \rho$ is expressed in terms of $\left[\bar{m}_{t}\left(m_{t}\right)\right]^{2}$, the QCD correction is only $(2-3) \cdot 10^{-3}$ in the next-to-leading-order approximation. As a consequence, when $\Delta \rho$ is rewritten in terms of $m_{t}^{2}$, the QCD correction to $\Delta \rho$ is almost entirely contained in $\left[\bar{m}_{t}\left(m_{t}\right) / m_{t}\right]^{2}$, i.e., it is a pure QCD effect. Finally, we observe that the $\mathcal{O}\left(\alpha_{s}^{2} G_{F} m_{t}^{2}\right)$ term indeed has the very sign predicted by the study [28,32,33] of the $t \bar{t}$ threshold effects and accounts also for the bulk of their size. In fact, this term may be absorbed into the $\mathcal{O}\left(\alpha_{s} G_{F} m_{t}^{2}\right)$ term by choosing $\xi=0.348$ for $N_{F}=6$ [18]. Arguing that $N_{F}=5$ is more appropriate for $\mu<m_{t}$, this value comes down to $\xi=0.324$ [42], which is not far outside the range $0.133 \leq \xi \leq 0.287$ predicted from the $t \bar{t}$ threshold analysis. The residual difference may be understood by observing that the ladder diagrams of $\mathcal{O}\left(\alpha_{s}^{n} G_{F} m_{t}^{2}\right)$, with $n \geq 3$, and the effect of the finite top-quark decay width are not included in the fixed-order calculation of Ref. 18.

The claim [43] that the $t \bar{t}$ threshold effects are greatly overestimated in Refs. [28, 32] is based on a simplified analysis, which demonstrably [33] suffers from a number of severe analytical and numerical errors. Speculations [44 that the dispersive computation of $t \bar{t}$ threshold effects is unstable are quite obviously unfounded, since they arise from uncorrelated and unjustifiably extreme variations of the continuum and threshold contributions. In particular, the authors of Ref. 44] ascribe the unavoidable scale dependence of the $\mathcal{O}\left(\alpha_{s} G_{F} m_{t}^{2}\right)$ continuum result to the uncertainty in the much smaller threshold contribution, which artificially amplifies this uncertainty. In fact, the sum of both contributions, which is the physically relevant quantity, is considerably less $\mu$ dependent than the continuum contribution alone [33.

\subsection{Specific Corrections: $\Gamma(Z \rightarrow b \bar{b})$ and $\Gamma(Z \rightarrow$ hadrons $)$}

In the so-called improved Born approximation $4, \overline{7}, \Gamma(Z \rightarrow f \bar{f})$ is given by the formula

$$
\Gamma(Z \rightarrow f \bar{f})=\frac{N_{f} G_{F} M_{Z}^{3}}{24 \pi \sqrt{2}} \rho\left[\left(1-4\left|Q_{f}\right| s_{w}^{2} \kappa\right)^{2} R^{V}+R^{A}\right]
$$

where $N_{f}=1$ (3) for lepton (quark) flavours, $\rho=(1-\Delta \rho)^{-1}$ and $\kappa=1+\Delta \kappa$, and $R^{V}$ and $R^{A}$ are the vector and axial-vector current correlators of $f$. Keeping the full $m_{f}$ dependence and including $\mathcal{O}(\alpha)$ electromagnetic corrections, the latter read [45]

$$
\begin{aligned}
& R^{V}=\left(1+\frac{1}{2 r}\right) \sqrt{1-\frac{1}{r}}+\frac{3 \alpha}{4 \pi} Q_{f}^{2}\left[1+\frac{3}{r}+\mathcal{O}\left(\frac{\ln r}{r^{2}}\right)\right], \\
& R^{A}=\left(1-\frac{1}{r}\right)^{3 / 2}+\frac{3 \alpha}{4 \pi} Q_{f}^{2}\left\{1+\frac{3}{r}\left[\ln (4 r)-\frac{1}{2}\right]+\mathcal{O}\left(\frac{\ln r}{r^{2}}\right)\right\},
\end{aligned}
$$

where $r=\left(M_{Z}^{2} / 4 m_{f}^{2}\right)$. The QCD corrections to $R^{V}$ and $R^{A}$, which are present if $f$ represents a quark, will be discussed in Sects. 2.2.3 and 2.2.4. The observable $\Gamma(Z \rightarrow b \bar{b})$ deserves special attention, since it receives specific $m_{t}$ power corrections. These may be accommodated 
in Eq. (11) by replacing $\rho$ and $\kappa$ with $\rho_{b}=\rho(1+\tau)^{2}$ and $\kappa_{b}=\kappa(1+\tau)^{-1}$, respectively, where $\tau$ is an additional electroweak parameter [46]. Similarly to $\Delta \rho, \tau$ receives contributions in the orders $G_{F} m_{t}^{2}, G_{F}^{2} m_{t}^{4}, \alpha_{s} G_{F} m_{t}^{2}$, etc. Equation (11) may be rendered exact by including in $\rho$ and $\kappa$ the subleading flavour-dependent weak corrections 46.

\subsubsection{Two-Loop $\mathcal{O}\left(G_{F}^{2} m_{t}^{4}\right)$ Corrections for $M_{H} \neq 0$}

In the oblique corrections considered so far, the $m_{t}$ dependence might be masked by all kinds of physics beyond the standard model. Contrariwise, in the case of $Z \rightarrow b \bar{b}$, the virtual top quark is tagged directly by the external bottom flavour. At one loop, there is a strong cancellation between the flavour-independent oblique corrections, $\Delta \rho$ and $\Delta \kappa$, and the specific $Z \rightarrow b \bar{b}$ vertex correction, $\tau$ 46.

Recently, the leading two-loop corrections to $\tau$, of $\mathcal{O}\left(G_{F}^{2} m_{t}^{4}\right)$ [20] and $\mathcal{O}\left(\alpha_{s} G_{F} m_{t}^{2}\right)$ [47], have become available. The master formula reads

$$
\tau=-2 x_{t}\left[1+x_{t} \tau^{(2)}\left(\frac{M_{H}}{m_{t}}\right)-2 \zeta(2) \frac{\alpha_{s}\left(m_{t}\right)}{\pi}\right],
$$

where $x_{t}$ is defined above Eq. (2). $\tau^{(2)}(r)$ [20] rapidly varies with $r, \tau^{(2)}(r) \geq \tau^{(2)}(1.55)=$ 1.23 , and its asymptotic behaviour is given by

$$
\tau^{(2)}(r)= \begin{cases}-2 \zeta(2)+9-4 \pi r+\mathcal{O}\left(r^{2} \ln r\right), & \text { if } r \ll 1 \\ \frac{5}{2} \ln ^{2} r-\frac{47}{12} \ln r+\zeta(2)+\frac{311}{144}+O\left(\frac{\ln ^{2} r}{r^{2}}\right), & \text { if } r \gg 1\end{cases}
$$

The value at $r=0$ has been confirmed by a third group 48.

\subsubsection{Two-Loop $\mathcal{O}\left(\alpha_{s} G_{F} m_{t}^{2}\right)$ Corrections}

In Eq. (13), we have also included the $\mathcal{O}\left(\alpha_{s} G_{F} m_{t}^{2}\right)$ term [47]. Notice that we have written it in a form appropriate for use in Eq. (11) together with the QCD-corrected expressions for $R^{V}$ and $R^{A}$, which have the common beginning $\left(1+\alpha_{s} / \pi\right)$ (see Sect. 2.2.3). We observe that the $\mathcal{O}\left(G_{F}^{2} m_{t}^{4}\right)$ and $\mathcal{O}\left(\alpha_{s} G_{F} m_{t}^{2}\right)$ terms of Eq. (13) cancel partially.

\subsubsection{Three-Loop $\mathcal{O}\left(\alpha_{s}^{3}\right)$ Corrections}

Most of the results discussed in this section are valid also for the $Z \rightarrow q \bar{q}$ decays with $q \neq b$. Here, we put $m_{q}=0$, except for $q=t$. Finite- $m_{b}$ effects will be considered in Sect. 2.2.4. By the optical theorem, the QCD corrections to $\Gamma(Z \rightarrow q \bar{q})$ may be viewed as the imaginary parts of the $Z$-boson self-energy diagrams that contain a $q$-quark loop decorated with virtual gluons and possibly other quark loops. Diagrams where the two $Z$ boson lines are linked to the same quark loop are usually called non-singlet, while the residual diagrams are called singlet, which includes the double-triangle diagrams. By $\gamma_{5}$ reflection, the non-singlet contribution, $R_{N S}$, to $R^{A}$ coincides with the one to $R^{V}$. Up to $\mathcal{O}\left(\alpha_{s}^{3}\right)$ in the $\overline{\mathrm{MS}}$ scheme with $N_{F}=5$ active flavours, one has

$$
R_{N S}=1+\frac{\alpha_{s}}{\pi}+\left(\frac{\alpha_{s}}{\pi}\right)^{2}\left[R_{1}+F\left(\frac{M_{Z}}{4 m_{t}^{2}}\right)\right]+R_{2}\left(\frac{\alpha_{s}}{\pi}\right)^{3}
$$


where $R_{1}=1.98571-0.11530 N_{F}=1.40923$ [49 and $R_{2}=-6.63694-1.20013 N_{F}-$ $0.00518 N_{F}^{2}=-12.76706$ [50]. $F$ collects the decoupling-top-quark effects in $\mathcal{O}\left(\alpha_{s}^{2}\right)$ and has the expansion 51

$$
F(r)=r\left[-\frac{8}{135} \ln (4 r)+\frac{176}{675}\right]+\mathcal{O}\left(r^{2}\right) .
$$

$F$ has also been obtained in numerical form recently [52]. We note that an analytic expression for $F$ had been known previously from the study of the two-loop QED vertex correction due to virtual heavy fermions [53] (see also Ref. [54]). Recently, the $\mathcal{O}\left(\alpha_{s}^{4}\right)$ term of Eq. (15) has been estimated using the principle of minimal sensitivity and the effective-charges approach [55]. The $\mathcal{O}\left(\alpha^{2}\right)$ and $\mathcal{O}\left(\alpha \alpha_{s}\right)$ corrections to $\Gamma(Z \rightarrow b \bar{b})$ from photonic source are well under control [56].

Due to Furry's theorem [57], singlet diagrams with $q \bar{q} Z$ vector couplings occur just in $\mathcal{O}\left(\alpha_{s}^{3}\right)$. They contain two quark loops at the same level of hierarchy, which, in general, involve different flavours. Thus, they cannot be assigned unambiguously to a specific $q \bar{q}$ channel. In practice, this does not create a problem, since their combined contribution to $\Gamma(Z \rightarrow$ hadrons $)$ is very small anyway [50],

$$
\delta \Gamma_{Z}=\frac{G_{F} M_{Z}^{3}}{8 \pi \sqrt{2}}\left(\sum_{q=u, d, s, c, b} v_{q}\right)^{2}(-0.41318)\left(\frac{\alpha_{s}}{\pi}\right)^{3},
$$

where $v_{q}=2 I_{q}-4 Q_{q} s_{w}^{2}$.

Axial-type singlet diagrams contribute already in $\mathcal{O}\left(\alpha_{s}^{2}\right)$. The sum over triangle subgraphs involving mass-degenerate (e.g., massless) up- and down-type quarks vanishes. Thus, after summation, only the double-triangle diagrams involving top and bottom quarks contribute to $\Gamma(Z \rightarrow b \bar{b})$ and $\Gamma(Z \rightarrow$ hadrons $)$. The present knowledge of the singlet part, $R_{S}^{A}$, of $R^{A}$ is summarized by

$$
R_{S}^{A}=\left(\frac{\alpha_{s}}{\pi}\right)^{2} \frac{1}{3} I\left(\frac{M_{Z}^{2}}{4 m_{t}^{2}}\right)+\left(\frac{\alpha_{s}}{\pi}\right)^{3}\left(\frac{23}{12} \ln ^{2} \frac{m_{t}^{2}}{M_{Z}^{2}}-\frac{67}{18} \ln \frac{m_{t}^{2}}{M_{Z}^{2}}-15.98773\right) .
$$

An analytic expression for the $I$ function may be found in Ref. [58]; its high- $m_{t}$ expansion reads [58

$$
I(r)=3 \ln (4 r)-\frac{37}{4}+\frac{28}{27} r+\mathcal{O}\left(r^{2}\right) .
$$

The second and third terms on the right-hand side of Eq. (19) have been confirmed recently in Refs. [59,60, respectively. The $\mathcal{O}\left(\alpha_{s}^{3}\right)$ logarithmic terms of Eq. (18) follow from Eq. (19) by means of renormalization-group techniques [59], while the constant term requires a separate computation [61]. Note that, in contrast to the formulations of Ref. [61], Eq. (18) is written in terms of the top-quark pole mass.

In $\mathcal{O}\left(\alpha_{s}^{3}\right), \Gamma(Z \rightarrow$ hadrons $)$ receives a contribution also from the $Z \rightarrow g g g$ decay, which proceeds through quark triangle and box diagrams. The decay mode $Z \rightarrow g g$ is prohibited by the Landau-Yang theorem [62]. The vector and axial-vector parts of $\Gamma(Z \rightarrow g g g)$ may be found in Refs. [63,64, respectively. Notice that these contributions are already contained in 
Eqs. (17, 18), respectively. Less than three out of one million hadronic $Z$-boson decays are due to the $Z \rightarrow g g g$ mechanism [64].

\subsubsection{Finite- $m_{b}$ Effects}

In $\mathcal{O}\left(\alpha_{s}\right)$, the full $m_{b}$ dependence of $R^{V}$ and $R^{A}$ is known [29,45], while, in higher orders, only the first terms of their $m_{b}^{2} / M_{Z}^{2}$ expansions have been calculated 66, 66, 67. In the $\overline{\mathrm{MS}}$ scheme with $N_{F}=5$, one has

$$
\begin{aligned}
\delta R^{V}= & 12 \frac{\alpha_{s}}{\pi} \frac{\bar{m}_{b}^{2}}{M_{Z}^{2}}\left[1+\frac{629}{72} \frac{\alpha_{s}}{\pi}+45.14610\left(\frac{\alpha_{s}}{\pi}\right)^{2}\right] \\
\delta R^{A}= & -6 \frac{\bar{m}_{b}^{2}}{M_{Z}^{2}}\left[1+\frac{11}{3} \frac{\alpha_{s}}{\pi}+\left(\frac{\alpha_{s}}{\pi}\right)^{2}\left(11.28560-\ln \frac{m_{t}^{2}}{M_{Z}^{2}}\right)\right] \\
& -\frac{10}{27}\left(\frac{\alpha_{s}}{\pi}\right)^{2} \frac{\bar{m}_{b}^{2}}{m_{t}^{2}}\left(\frac{8}{3}+\frac{1}{2} \ln \frac{m_{t}^{2}}{M_{Z}^{2}}\right),
\end{aligned}
$$

where $\alpha_{s}$ and the bottom-quark $\overline{\mathrm{MS}}$ mass, $\bar{m}_{b}$, are to be evaluated at $\mu=M_{Z}$. Due to the use of $\bar{m}_{b}\left(M_{Z}\right)$ instead of $m_{b}$, Eqs. (20,21) are devoid of terms involving $\ln \left(M_{Z}^{2} / m_{b}^{2}\right)$. The second and third terms of Eq. (20) come from Refs. 65, 66, I respectively, and the $\mathcal{O}\left(\alpha_{s}^{2}\right)$ terms in the first and second lines of Eq. (21) are from Refs. 67,60, respectively. The $\mathcal{O}\left(\alpha_{s}^{3} \bar{m}_{b}^{2} / M_{Z}^{2}\right)$ term of $R^{A}$ has not yet appeared in the literature. The $\mathcal{O}\left(\alpha_{s} m_{b}^{2} / M_{Z}^{2}\right)$ corrections should be detectable. For $m_{t}=174 \mathrm{GeV}$, the finite- $m_{b}$ terms beyond $\mathcal{O}\left(\alpha_{s}\right)$ in Eqs. $(20,21)$ each amount to approximately $5 \cdot 10^{-3} \%$ of $\Gamma(Z \rightarrow b \bar{b})$ but have opposite signs. As might be expected, the $\mathcal{O}\left(\alpha_{s}^{2} \bar{m}_{b}^{2} / m_{t}^{2}\right)$ term is insignificant numerically, reducing $\Gamma(Z \rightarrow b \bar{b})$ by $3 \cdot 10^{-5 \%}$.

Note that Eqs. $(20,21)$ do not include the contribution due to the $Z \rightarrow q \bar{q} b \bar{b}$ decays, 2 where a bottom-quark pair is radiated off via a virtual gluon from a primary $q$-quark line, with $q=u, d, s, c$. These contributions are usually ascribed to the $\mathcal{O}\left(\alpha_{s}^{2}\right)$ corrections to $\Gamma(Z \rightarrow q \bar{q})$. Under favourable experimental conditions, these events can be tagged by their $b \bar{b}$ invariant mass, which is typically low. A closed expression for $\Gamma(Z \rightarrow q \bar{q} b \bar{b})$ in the approximation $m_{q}=0$ may be found in Ref. [54]. By including also the $q \bar{q} Z$ vertex correction induced by a virtual bottom quark [53], one obtains the corresponding $\mathcal{O}\left(\alpha_{s}^{2}\right)$ contribution to $R_{N S}$ in the $q \bar{q}$ channel. It has the expansion [54] (see also Ref. [52])

$$
\delta R_{N S}=\left(\frac{\alpha_{s}}{\pi}\right)^{2}\left\{\frac{2}{3} \zeta(3)-\frac{11}{12}+x^{2}\left[-\ln x-4 \zeta(3)+\frac{13}{3}\right]+\mathcal{O}\left(x^{3}\right)\right\}
$$

where $x=m_{b}^{2} / M_{Z}^{2}$. The first term on the right-hand side of Eq. (22) coincides with the coefficient of the $N_{F}$-dependent term of $R_{1}$ in Eq. (15). Notice that the logarithmic term in

\footnotetext{
${ }^{1}$ There is an obvious typographical error on the right-hand side of the last equation of Eq. (23) in Ref. [66]: 92 should be replaced by 96 . Furthermore, the coefficient of $\zeta(3)$ in the expression for $b_{3}$ should read $992 / 72$ instead of $1008 / 72$ 68.

${ }^{2}$ It is justified to distinguish between the $Z \rightarrow q \bar{q} b \bar{b}$ and $Z \rightarrow b \bar{b} q \bar{q}$ decays, since the interference of the respective sets of diagrams vanishes upon summation over isodoublets of $q$ quarks [58].
} 
Eq. (22) cannot be eliminated by introducing $\bar{m}_{b}\left(M_{Z}\right)$. Due to the absence of a term linear in $x$, the finite- $m_{b}$ corrections in Eq. (22) will be difficult to probe experimentally.

For the reader's convenience, we recall the procedure of evaluating $\bar{m}_{b}(\mu)$. For later use in Sect. 3.2.1, we consider also charm quarks. The quark pole masses, $m_{q}$, may be estimated from QCD sum rules [69]. Recent analyses [70] have yielded $m_{c}=(1.46 \pm 0.07) \mathrm{GeV}$ and $m_{b}=(4.72 \pm 0.05) \mathrm{GeV}$. To obtain $\bar{m}_{q}(\mu)$, one proceeds in two steps. Firstly, one evaluates $\bar{m}_{q}\left(m_{q}\right)$ from

$$
\frac{m_{q}}{\bar{m}_{q}\left(m_{q}\right)}=1+C_{F} \frac{\alpha_{s}\left(m_{q}\right)}{\pi}+K_{q}\left[\frac{\alpha_{s}\left(m_{q}\right)}{\pi}\right]^{2},
$$

with 40,41

$$
K_{q} \approx 16.11006-1.04137 \sum_{i=1}^{N_{F}-1}\left(1-\frac{4}{3} \frac{m_{i}}{m_{q}}\right),
$$

where the sum extents over all quark flavours with $m_{i}<m_{q}$. Assuming $m_{s}=0.266 \mathrm{GeV}$ 71] and neglecting $m_{u}$ and $m_{d}$, one finds $K_{c}=13.2$ and $K_{b}=12.5$. Secondly, one determines $\bar{m}_{q}(\mu)$ via the scaling law

$$
\bar{m}_{q}(\mu)=\bar{m}_{q}\left(m_{q}\right) \frac{c_{q}\left(\alpha_{s}(\mu) / \pi\right)}{c_{q}\left(\alpha_{s}\left(m_{q}\right) / \pi\right)}
$$

where 40,72]

$$
\begin{aligned}
& c_{c}(x)=\left(\frac{25}{6} x\right)^{12 / 25}\left(1+1.01413 x+1.38921 x^{2}\right) \\
& c_{b}(x)=\left(\frac{23}{6} x\right)^{12 / 23}\left(1+1.17549 x+1.50071 x^{2}\right) .
\end{aligned}
$$

\section{Higgs Sector: Corrections to $\Gamma(H \rightarrow f \bar{f})$ and $\Gamma(H \rightarrow$ hadrons)}

Quantum corrections to Higgs-boson phenomenology have received much attention in the literature; for a review, see Ref. [8]. The experimental relevance of radiative corrections to the $f \bar{f}$ branching fractions of the Higgs boson has been emphasized recently in the context of a study dedicated to LEP 2 [73]. Techniques for the measurement of these branching fractions at a $\sqrt{s}=500 \mathrm{GeV} e^{+} e^{-}$linear collider have been elaborated in Ref. [74.

In the Born approximation, the $f \bar{f}$ partial widths of the Higgs boson are given by 75

$$
\Gamma_{0}(H \rightarrow f \bar{f})=\frac{N_{f} G_{F} M_{H} m_{f}^{2}}{4 \pi \sqrt{2}}\left(1-\frac{4 m_{f}^{2}}{M_{H}^{2}}\right)^{3 / 2} .
$$

The full one-loop electroweak corrections to Eq. (27) are now well established [76, 77,. They consist of an electromagnetic and a weak part, which are separately finite and gauge independent. They may be included in Eq. (27) as an overall factor, $\left[1+(\alpha / \pi) Q_{f}^{2} \Delta_{e m}\right]\left(1+\Delta_{\text {weak }}\right)$. 
For $M_{H} \gg 2 m_{f}, \Delta_{e m}$ develops a large logarithm,

$$
\Delta_{e m}=-\frac{3}{2} \ln \frac{M_{H}^{2}}{m_{f}^{2}}+\frac{9}{4}+\mathcal{O}\left(\frac{m_{f}^{2}}{M_{H}^{2}} \ln \frac{M_{H}^{2}}{m_{f}^{2}}\right) .
$$

For $M_{H} \ll 2 M_{W}$, the weak part is well approximated by 77

$$
\Delta_{\text {weak }}=\frac{G_{F}}{8 \pi^{2} \sqrt{2}}\left\{C_{f} m_{t}^{2}+M_{W}^{2}\left(\frac{3}{s_{w}^{2}} \ln c_{w}^{2}-5\right)+M_{Z}^{2}\left[\frac{1}{2}-3\left(1-4 s_{w}^{2}\left|Q_{f}\right|\right)^{2}\right]\right\},
$$

where $C_{b}=1$ and $C_{f}=7$ for all other flavours, except for top. The $t \bar{t}$ mode will not be probed experimentally anytime soon and we shall not be concerned with it in the remainder of this article. Equation (29) has been obtained by putting $M_{H}=m_{f}=0(f \neq t)$ in the expression for the full one-loop weak correction. It provides a very good approximation for $f=\tau$ up to $M_{H} \approx 135 \mathrm{GeV}$ and for $f=b$ up to $M_{H} \approx 70 \mathrm{GeV}$, the relative deviation from the full weak correction being less than $15 \%$ in each case.

\subsection{Two-Loop $\mathcal{O}\left(\alpha_{s} G_{F} m_{t}^{2}\right)$ Corrections to $\Gamma(H \rightarrow f \bar{f})$}

From Eq. (29) it is evident that the dominant effect is due to virtual top quarks. In the case $f \neq b$, the $m_{t}$ dependence is carried solely by the renormalizations of the wave function and the vacuum expectation value of the Higgs field and is thus flavour independent. These corrections are of the same nature as those considered in Ref. [78]. For $f=b$, there are additional $m_{t}$-dependent contributions from the $b \bar{b} H$ vertex correction and the bottom-quark wave-function renormalization. Incidentally, they cancel almost completely the universal $m_{t}$ dependence. It is amusing to observe that a similar situation has been encountered in the context of the $Z \rightarrow f \bar{f}$ decays [46]. The QCD corrections to the universal and non-universal $\mathcal{O}\left(G_{F} m_{t}^{2}\right)$ terms will be presented in Sects. 3.1.1 and 3.1.2, respectively.

\subsubsection{Universal Corrections}

The universal $\mathcal{O}\left(G_{F} m_{t}^{2}\right)$ term of $\Delta_{\text {weak }}$ resides inside the combination

$$
\Delta_{u}=-\frac{\Pi_{W W}(0)}{M_{W}^{2}}-\operatorname{Re} \Pi_{H H}^{\prime}\left(M_{H}^{2}\right),
$$

where $\Pi_{W W}$ and $\Pi_{H H}$ are the unrenormalized self-energies of the $W$ and Higgs bosons, respectively [77]. The same is true of its QCD correction.

For $M_{H}<2 m_{t}$ and $m_{b}=0$, the one-loop term reads [77]

$$
\Delta_{u}^{0}=4 N_{c} x_{t}\left[\left(1+\frac{1}{2 r}\right) \sqrt{\frac{1}{r}-1} \arcsin \sqrt{r}-\frac{1}{4}-\frac{1}{2 r}\right],
$$

where $r=\left(M_{H}^{2} / 4 m_{t}^{2}\right)$ and $x_{t}$ is defined above Eq. (2). In the same approximation, the two-loop term may be written as [30,89]

$$
\Delta_{u}^{1}=N_{c} C_{F} x_{t} \frac{\alpha_{s}}{\pi}\left[6 \zeta(3)+2 \zeta(2)-\frac{19}{4}-\operatorname{Re} H_{1}^{\prime}(r)\right],
$$


where $C_{F}=\left(N_{c}^{2}-1\right) /\left(2 N_{c}\right)=4 / 3$ and $H_{1}$ has an expression in terms of dilogarithms and trilogarithms [79]. Equation (32]) has been confirmed recently [80]. In the heavy-quark limit $(r \ll 1)$, one has 79

$$
H_{1}^{\prime}(r)=6 \zeta(3)+3 \zeta(2)-\frac{13}{4}+\frac{122}{135} r+\mathcal{O}\left(r^{2}\right) .
$$

Combining Eqs. (31,32) and retaining only the leading high- $m_{t}$ terms, one finds the QCDcorrected coefficients $C_{f}$ for $f \neq b$,

$$
C_{f}=7-2\left(\frac{\pi}{3}+\frac{3}{\pi}\right) \alpha_{s} \approx 7-4.00425 \alpha_{s}
$$

This result has been reproduced recently [81]. We recover the notion that, in electroweak physics, the one-loop $\mathcal{O}\left(G_{F} m_{t}^{2}\right)$ terms get screened by their QCD corrections. The QCD correction to the shift in $\Gamma(H \rightarrow f \bar{f})$ induced by a pair of novel quarks with arbitrary masses may be found in Ref. [79].

\subsubsection{Non-universal Corrections}

The QCD correction to the non-universal one-loop contribution to $\Gamma(H \rightarrow b \bar{b})$ arises in part from genuine two-loop three-point diagrams, which are more involved technically. However, the leading high- $m_{t}$ term may be extracted [82] by means of a low-energy theorem [83], which relates the amplitudes of two processes that differ by the insertion of an external Higgs-boson line carrying zero momentum. In this way, one only needs to compute the irreducible two-loop bottom-quark self-energy diagrams with one gluon and one longitudinal $W$ boson, which may be taken massless. After using the Dirac equation and factoring out one power of $m_{b}$, one may put $m_{b}=0$ in the two-loop integrals, which may then be solved analytically. Applying the low-energy theorem and performing on-shell renormalization, one eventually finds the non-universal leading high- $m_{t}$ term along with its QCD correction [82],

$$
\Delta_{n u}=x_{t}\left(-6+\frac{3}{2} C_{F} \frac{\alpha_{s}}{\pi}\right)
$$

Combining the term contained within the parentheses with Eq. (34), one obtains the QCDcorrected coefficient $C_{b}$,

$$
C_{b}=1-2\left(\frac{\pi}{3}+\frac{2}{\pi}\right) \alpha_{s} \approx 1-3.36763 \alpha_{s}
$$

Again, the $\mathcal{O}\left(G_{F} m_{t}^{2}\right)$ term is screened by its QCD correction.

\subsection{QCD Corrections to $\Gamma(H \rightarrow$ hadrons $)$}

Both $H \rightarrow q \bar{q}$ and $H \rightarrow g g$ lead to two-jet final states. These processes are relevant phenomenologically for the search for the low- and intermediate-mass Higgs boson, with $M_{H}<2 M_{W}$, at $e^{+} e^{-}$colliders, while they are very difficult to discern from background 
reactions at hadron colliders. The partial widths of both decay modes receive significant QCD corrections.

\subsubsection{Two-Loop $\mathcal{O}\left(\alpha_{s}^{2}\right)$ Corrections to $\Gamma(H \rightarrow q \bar{q})$ Including Finite- $m_{q}$ Effects}

In the on-shell scheme, the one-loop QCD correction [84 to $\Gamma(H \rightarrow q \bar{q})$ emerges from one-loop QED correction by substituting $\alpha_{s} C_{F}$ for $\alpha Q_{f}^{2}$. From Eq. (28) it is apparent that, for $m_{q} \ll M_{H} / 2$, large logarithmic corrections occur. In general, they are of the form $\left(\alpha_{s} / \pi\right)^{n} \ln ^{m}\left(M_{H}^{2} / m_{q}^{2}\right)$, with $n \geq m$. Owing to the renormalization-group equation, these logarithms may be absorbed completely into the running $\overline{\mathrm{MS}}$ quark mass, $\bar{m}_{q}(\mu)$, evaluated at $\mu=M_{H}$. A similar mechanism has been exploited also in Eqs. (20,21). In this way, these logarithms are resummed to all orders and the perturbation expansion converges more rapidly. This observation gives support to the notion that the $q \bar{q} H$ Yukawa couplings are controlled by the running quark masses.

For $q \neq t$, the QCD corrections to $\Gamma(H \rightarrow q \bar{q})$ are known up to $\mathcal{O}\left(\alpha_{s}^{2}\right)$. In the $\overline{\mathrm{MS}}$ scheme, the result is 8

$$
\begin{aligned}
\Gamma(H \rightarrow q \bar{q})=\frac{3 G_{F}}{4} M_{H} \bar{m}_{q}^{2} & \multicolumn{2}{c}{\left(1-4 \frac{\bar{m}_{q}^{2}}{M_{H}^{2}}\right)^{3 / 2}+C_{F} \frac{\alpha_{s}}{\pi}\left(\frac{17}{4}-30 \frac{\bar{m}_{q}^{2}}{M_{H}^{2}}\right)} \\
+ & \left(\frac{\alpha_{s}}{\pi}\right)^{2}\left[K_{1}+K_{2} \frac{\bar{m}_{q}^{2}}{M_{H}^{2}}+12 \sum_{i=u, d, s, c, b} \frac{\bar{m}_{i}^{2}}{M_{H}^{2}}\right. \\
+ & \left.\left.\frac{1}{3}\left(\frac{1}{3} \ln ^{2} \frac{M_{H}^{2}}{\bar{m}_{q}^{2}}-2 \ln \frac{M_{H}^{2}}{m_{t}^{2}}+8-2 \zeta(2)\right)\right]\right\},
\end{aligned}
$$

where $K_{1}=35.93996-1.35865 N_{F}$ [72], $K_{2}=-129.72924+6.00093 N_{F}$ [41], with $N_{F}$ being the number of quark flavours active at $\mu=M_{H}$, and it is understood that $\alpha_{s}, \bar{m}_{q}$, and $\bar{m}_{i}$ are to be evaluated at this scale. The last term in the second line of Eq. (37) has been found in Ref. [41], too. The term in the third line is related to the double-triangle diagram involving the $q$ and top quarks [85]; its logarithmic dependence on $\bar{m}_{q}$ is compensated in the Higgs-boson hadronic width by a similar term connected with the $g g$ final state [85]. Equation (37) may be translated into the on-shell scheme by using the relation between $m_{q}$ and $\bar{m}_{q}\left(M_{H}\right)$ (see Sect. 2.2.4) [86]. The difference between these two evaluations is extremely small [86], which indicates that the residual uncertainty due to the lack of knowledge of the $\mathcal{O}\left(\alpha_{s}^{3}\right)$ term is likely to be inconsequential for practical purposes.

The electroweak corrections may be implemented in Eq. (37) by multiplication with $\left[1+(\alpha / \pi) Q_{f}^{2} \Delta_{e m}\right]\left(1+\Delta_{w e a k}\right)$, where $\Delta_{e m}$ and $\Delta_{\text {weak }}$ are given in Eqs. (28,29), respectively. To include also the $\mathcal{O}\left(\alpha_{s} G_{F} m_{t}^{2}\right)$ corrections, one substitutes in Eq. (29) the QCD-corrected $C_{f}$ terms specified in Eqs. (34,36). We note in passing that our result [82 disagrees with a recent calculation 81] of the $\mathcal{O}\left(\alpha_{s} G_{F} m_{t}^{2}\right)$ correction to $\Gamma(H \rightarrow b \bar{b})$ in the on-shell scheme.

\subsubsection{Two-Loop $\mathcal{O}\left(\alpha_{s}\right)$ Corrections to $\Gamma(H \rightarrow g g)$}

The hadronic width of the Higgs boson receives contributions also from the $H \rightarrow g g$ channel, which is mediated by massive-quark triangle diagrams. To lowest order, $\Gamma(H \rightarrow g g)$ 
[87] is well approximated by [8]

$$
\Gamma(H \rightarrow g g)=\frac{\alpha_{s}^{2} G_{F} M_{H}^{3}}{36 \pi^{3} \sqrt{2}}\left(1+\frac{7}{60} \frac{M_{H}^{2}}{m_{t}^{2}}\right) .
$$

The next-to-leading-order QCD correction may be accommodated in Eq. (38) by multiplication with 88

$$
\left[1+\frac{\alpha_{s}\left(M_{H}\right)}{\pi}\left(\frac{95}{4}-\frac{7}{6} N_{F}\right)\right],
$$

where $N_{F}$ is the number of quark flavours active at scale $M_{H}$. Equation (39) includes twoloop corrections in the $g g$ channel and one-loop contributions due to the $g g g$ and $g q \bar{q}$ modes, where the $q \bar{q}$ pair is created from a virtual gluon. Assuming $N_{F}=5$ and $\alpha_{s}\left(M_{H}\right)=0.11$, Eq. (39) is as large as 1.63. For $M_{H}<2 M_{H}$, the two-loop QCD correction to $\Gamma(H \rightarrow \gamma \gamma)$ is below $3 \%$ in size [89] and the one to $\Gamma(H \rightarrow Z \gamma)$ below $0.3 \%$ [90.

\section{Conclusions}

In conclusion, all dominant two-loop and even certain three-loop radiative corrections to $Z$ boson physics are now available. However, one has to bear in mind that, apart from the lack of knowledge of the accurate values of $M_{H}, m_{t}$, and $\alpha_{s}\left(M_{Z}\right)$, the reliability of the theoretical predictions is limited by a number of error sources. The inherent QCD errors on the hadronic contribution to $\Delta \alpha$ and the $t b$ contribution to $\Delta \rho$ are $\delta \Delta \alpha= \pm 7 \cdot 10^{-4}$ and $\delta \Delta \rho= \pm 1.5 \cdot 10^{-4}$, respectively, which amounts to $\delta \Delta r= \pm 8.6 \cdot 10^{-4}$. The unknown electroweak corrections are of the order $\left(\alpha / \pi s_{w}^{2}\right)^{2}\left(m_{t}^{2} / M_{Z}^{2}\right) \ln \left(m_{t}^{2} / M_{Z}^{2}\right) \approx 6 \cdot 10^{-4}$, possibly multiplied by a large prefactor [21]. The scheme dependence of the key electroweak parameters has been estimated in Refs. 32,91,92 by comparing the evaluations in the on-shell scheme and certain variants of the $\overline{\mathrm{MS}}$ scheme; the maximum variation of $\Delta r$ in the ranges $60 \mathrm{GeV} \leq M_{H} \leq 1 \mathrm{TeV}$ and $150 \mathrm{GeV} \leq m_{t} \leq 200 \mathrm{GeV}$ is $8 \cdot 10^{-5}$ when the coupling-constant renormalization

is converted [32 and $4 \cdot 10^{-4}$ when the top-quark mass is redefined taking into account just the QCD corrections [91]. The effect on $\Delta \rho$ of including also the leading electroweak corrections in the redefinition of the top-quark mass has been investigated recently in the approximation $M_{H}, m_{t} \gg M_{Z}$ [92]. The theoretical predictions for Higgs-boson physics at present and near-future colliding-beam experiments are probably far more precise than the expected theoretical errors.

\section{ACKNOWLEDGMENTS}

I would like to thank Jochem Fleischer, Hans Kühn, Alberto Sirlin, and Levan Surguladze for clarifying comments regarding Refs. [18], [54,66, 67, [37, 38], and [65, respectively. I am grateful to the KEK Theory Group for the warm hospitality extended to me during my visit, when this review article was written. This work was supported by the Japan Society for the Promotion of Science (JSPS) through Fellowship No. S94159. 


\section{References}

[1] S.L. Glashow, Nucl. Phys. 22 (1961) 579; S. Weinberg, Phys. Rev. Lett. 19 (1967) 1264; A. Salam, in Elementary Particle Theory, ed. by N. Svartholm (Almqvist and Wiksell, Stockholm, 1968), p. 367.

[2] A. Sirlin, in Proceedings of the International Workshop on Supersymmetry and Unification of Fundamental Interactions (SUSY 93), Boston, Massachusetts, March 29-April 1, 1993, ed. by P. Nath (World Scientific, Singapore, 1993); B.A. Kniehl, in Proceedings of the International Europhysics Conference on High Energy Physics, Marseille, France, July 22-28, 1993, ed. by J. Carr and M. Perrottet (Editions Frontières, Gifsur-Yvette), p. 639; W. Hollik, in Proceedings of the XVI International Symposium on Lepton and Photon Interactions, Ithaca, New York, August 10-15, 1993, ed. by P. Drell and D. Rubin (AIP Press, New York, 1994), p. 352; P. Langacker, University of Pennsylvania Report No. UPR-0624T, hep-ph/9408310 (August 1994), invited talk presented at 22nd INS International Symposium on Physics with High Energy Colliders, Tokyo, Japan, March 8-10, 1994; J.L. Rosner, Enrico Fermi Institute Report No. EFI 94-38, hep-ph/9408349 (August 1994), to appear in Proceedings of the DPF 94 Meeting, Albuquerque, New Mexico, August 2-6, 1994 (World Scientific, Singapore, 1994); G. Altarelli, J. Fleischer, W. Hollik, and B.A. Kniehl, to appear in Proceedings of the Tennessee International Symposium on Radiative Corrections: Status and Outlook, Gatlinburg, Tennessee, June 27-July 1, 1994, ed. by B.F.L. Ward (World Scientific, Singapore, 1994).

[3] M.E. Peskin, in Proceedings of the Seventeenth SLAC Summer Institute on Particle Physics: Physics at the $100 \mathrm{GeV}$ Scale, July 10-21, 1989, ed. by E.C. Brennan, SLACReport-361 (January 1990), p. 71; F. Jegerlehner, in Progress in Particle and Nuclear Physics, Vol. 27, ed. by A. Faessler (Pergamon Press, Oxford, 1991), p. 1; W.J. Marciano, Annu. Rev. Nucl. Part. Sci. 41 (1991) 469; Z. Hioki, Mod. Phys. Lett. A7 (1992) 1009; W. Hollik, in Advanced Series on Directions in High Energy Physics - Vol. 10: Heavy Flavours, ed. by A.J. Buras and M. Lindner (World Scientific, Singapore, 1994), p. 1; W. Hollik and A. Sirlin, to appear in Advanced Series on Directions in High Energy Physics: Precision Tests of the Standard Electroweak Model, ed. by P. Langacker (World Scientific, Singapore, 1994).

[4] M. Consoli, W. Hollik, and F. Jegerlehner, in Z Physics at LEP 1, ed. by G. Altarelli, R. Kleiss, and C. Verzegnassi, CERN Yellow Report No. 89-08 (1989) Vol. 1, p. 7; W.F.L. Hollik, Fortschr. Phys. 38 (1990) 165.

[5] G. Burgers, F. Jegerlehner, B. Kniehl, and J. Kühn, in Z Physics at LEP 1, ed. by G. Altarelli, R. Kleiss, and C. Verzegnassi, CERN Yellow Report No. 89-08 (1989) Vol. 1, p. 55 . 
[6] F. Jegerlehner, in Testing the Standard Model-Proceedings of the 1990 Theoretical Advanced Study Institute in Elementary Particle Physics, ed. by M. Cvetič and P. Langacker (World Scientific, Singapore, 1991), p. 476.

[7] F. Halzen, B.A. Kniehl, and M.L. Stong, in Particle Physics: VI Jorge André Swieca Summer School, Campos de Jordão, Brasil, 14-26 January, 1991, ed. by O.J.P. Éboli, M. Gomes, and A. Santoro (World Scientific, Singapore, 1992), p. 219; Z. Phys. C58 (1993) 119.

[8] B.A. Kniehl, Phys. Rep. 240 (1994) 211.

[9] D.A. Ross and M. Veltman, Nucl. Phys. B95 (1975) 135; M. Veltman, Nucl. Phys. B123 (1977) 89.

[10] A. Sirlin, Phys. Rev. D22 (1980) 971.

[11] K-I. Aoki, Z. Hioki, R. Kawabe, M. Konuma, and T. Muta, Prog. Theor. Phys. 64 (1980) 707; Prog. Theor. Phys. 65 (1981) 1001; Prog. Theor. Phys. Suppl. 73 (1982) 1; M. Böhm, H. Spiesberger, and W. Hollik, Fortschr. Phys. 34 (1986) 687; A. Denner, Fortschr. Phys. 41 (1993) 307.

[12] B. Holdom and J. Terning, Phys. Lett. B247 (1990) 88; M.E. Peskin and T. Takeuchi, Phys. Rev. Lett. 65 (1990) 964; Phys. Rev. D46 (1992) 381; W.J. Marciano and J.L. Rosner, Phys. Rev. Lett. 65 (1990) 2963; D.C. Kennedy and P. Langacker, Phys. Rev. Lett. 65 (1990) 2967; Phys. Rev. D44 (1991) 1591; G. Altarelli and R. Barbieri, Phys. Lett. B253 (1991) 161; M. Golden and L. Randall, Nucl. Phys. B361 (1991) 3; E. Gates and J. Terning. Phys. Rev. Lett. 67 (1991) 1840; G. Altarelli, R. Barbieri, and S. Jadach, Nucl. Phys. B369 (1992) 3; G. Bhattacharyya, S. Banerjee, and P. Roy, Phys. Rev. D45 (1992) R729; F. Halzen, P. Roy, and M.L. Stong, Phys. Lett. B277 (1992) 503; E. Ma and P. Roy, Phys. Rev. Lett. 68 (1992) 2879; J. Ellis, G.L Fogli, and E. Lisi, Phys. Lett. B285 (1992) 238; ibid. B292 (1992) 427; T. Inami, C.S. Lim, and A. Yamada, Mod. Phys. Lett. A7 (1992) 2789; B.A. Kniehl and H.-G. Kohrs, Phys. Rev. D48 (1993) 225; S. Cortese, E. Pallante, and R. Petronzio, Phys. Lett. B301 (1993) 203; G. Altarelli, R. Barbieri, and F. Caravaglios, Nucl. Phys. B405 (1993) 3.

[13] G. Degrassi, B.A. Kniehl, and A. Sirlin, Phys. Rev. D48 (1993) R3963.

[14] J. van der Bij and M. Veltman, Nucl. Phys. B231 (1984) 205; J.J. van der Bij, Nucl. Phys. B248 (1984) 141.

[15] R. Barbieri, P. Ciafaloni, and A. Strumia, Phys. Lett. B317 (1993) 381.

[16] A. Djouadi and C. Verzegnassi, Phys. Lett. B195 (1987) 265; A. Djouadi, Nuovo Cim. 100A (1988) 357; A. Djouadi and P. Gambino, Phys. Rev. D49 (1994) 3499; ibid. D49 (1994) 4705. 
[17] B.A. Kniehl, J.H. Kühn, and R.G. Stuart, Phys. Lett. B214 (1988) 621; B.A. Kniehl, Nucl. Phys. B347 (1990) 86; F. Halzen and B.A. Kniehl, Nucl. Phys. B353 (1991) 567; B.A. Kniehl and R.G. Stuart, Comput. Phys. Commun. 72 (1992) 175.

[18] L. Avdeev, J. Fleischer, S. Mikhailov, and O. Tarasov, Bielfeld University Report No. BI-TP-93/60, hep-ph/9406363 (June 1994).

[19] A. Anselm, N. Dombey, and E. Leader, Phys. Lett. B312 (1993) 232.

[20] R. Barbieri, M. Beccaria, P. Ciafaloni, G. Curci, and A. Viceré, Phys. Lett. B288 (1992) 95; (E) B312 (1993) 511; Nucl. Phys. B409 (1993) 105; J. Fleischer, O.V. Tarasov, and F. Jegerlehner, Phys. Lett. B319 (1994) 249.

[21] G. Degrassi, S. Fanchiotti, and P. Gambino, Report No. CERN-TH.7180/94, DFPD 94/TH/12, and NYU-Th-94/02/01, hep-ph/9403250 (February 1994).

[22] J.J. van der Bij and F. Hoogeveen, Nucl. Phys. B283 (1987) 477; M. Consoli, W. Hollik, and F. Jegerlehner, Phys. Lett. B227 (1989) 167.

[23] K. Hikasa et al. (Particle Data Group), Phys. Rev. D45 (1992) S1.

[24] F. Jegerlehner, private communication.

[25] A.J. Weinstein and R. Stroynowski, Ann. Rev. Nucl. Part. Phys. 43 (1993) 457.

[26] R.E. Cutkosky, J. Math. Phys. 1 (1960) 429.

[27] B.A. Kniehl and A. Sirlin, Nucl. Phys. B371 (1992) 141.

[28] B.A. Kniehl and A. Sirlin, Phys. Rev. D47 (1993) 883.

[29] T.H. Chang, K.J.F. Gaemers, and W.L. van Neerven, Phys. Lett. 108B (1982) 222; Nucl. Phys. B202 (1982) 407.

[30] B.A. Kniehl and A. Sirlin, Phys. Lett. B318 (1993) 367.

[31] T. Takeuchi, A.K. Grant, and M.P. Worah, Report No. FERMILAB-PUB-94/172-T and EFI 94-32, hep-ph/9407349 (July 1994). Note, however, that there is a previous report by the same authors, Report No. FERMILAB-PUB-94/037-T and EFI 94-07, hep-ph/9403294 (March 1994), where it is argued, again appealing to the operator product expansion, that the dispersion relations of Refs. [27,29] are equivalent.

[32] S. Fanchiotti, B. Kniehl, and A. Sirlin, Phys. Rev. D48 (1993) 307.

[33] B.A. Kniehl and A. Sirlin, Report No. DESY 93-194 and NYU-Th-93/12/01, hepph/9401243 (December 1993), Phys. Rev. D (to appear).

[34] F. Abe et al. (CDF Collaboration), Phys. Rev. Lett. 73 (1994) 225; Phys. Rev. D50 (1994) 2966. 
[35] S. Bethke, to appear in Proceedings of the Tennessee International Symposium on Radiative Corrections: Status and Outlook, Gatlinburg, Tennessee, June 27-July 1, 1994, ed. by B.F.L. Ward (World Scientific, Singapore, 1994).

[36] B.H. Smith and M.B. Voloshin, Report No. UMN-TH-1241/94 and TPI-MINN-94/5-T, hep-ph/9401357 (January 1994).

[37] A. Sirlin, BNL Report No. BNL-60161, hep-ph/9403282 (March 1994).

[38] A. Sirlin, NYU Report No. NYU-TH-94/08/01, hep-ph/9409303 (August 1994).

[39] S.J. Brodsky, G.P. Lepage, and P.B. Mackenzie, Phys. Rev. D28 (1983) 228.

[40] N. Gray, D.J. Broadhurst, W. Grafe, and K. Schilcher, Z. Phys. C48 (1990) 673; K. Schilcher and L.R. Surguladze, private communications.

[41] L.R. Surguladze, University of Oregon Report No. OITS 543, hep-ph/9405325 (May 1994).

[42] J. Fleischer, private communication.

[43] F.J. Ynduráin, Phys. Lett. B321 (1994) 400.

[44] M.C. Gonzalez-Garcia, F. Halzen, and R.A. Vázquez, Phys. Lett. B322 (1994) 233.

[45] K. Schilcher, M.D. Tran, and N.F. Nasrallah, Nucl. Phys. B181 (1981) 91; (E) B187 (1981) 594; L.J. Reinders, H.R. Rubinstein, and S. Yazaki, Nucl. Phys. B186 (1981) 109; Phys. Lett. 103B (1981) 63; J. Jersák, E. Laermann, and P.M. Zerwas, Phys. Rev. D25 (1982) 1218; (E) D36 (1987) 310; A. Djouadi, J.H. Kühn, and P.M. Zerwas, Z. Phys. C46 (1990) 411.

[46] A.A. Akhundov, D.Yu. Bardin, and T. Riemann, Nucl. Phys. B276 (1986) 1; J. Bernabéu, A. Pich, and A. Santamaría, Phys. Lett. B200 (1988) 569; Nucl. Phys. B363 (1991) 326; W. Beenakker and W. Hollik, Z. Phys. C40 (1988) 141; B.W. Lynn and R.G. Stuart, Phys. Lett. B252 (1990) 676.

[47] J. Fleischer, O.V. Tarasov, F. Jegerlehner, and P. Rączka, Phys. Lett. B293 (1992) 437; G. Buchalla and A.J. Buras, Nucl. Phys. B398 (1993) 285; G. Degrassi, Nucl. Phys. B407 (1993) 271; K.G. Chetyrkin, A. Kwiatkowski, and M. Steinhauser, Mod. Phys. Lett. A8 (1993) 2785.

[48] A. Denner, W. Hollik, and B. Lampe, Z. Phys. C60 (1993) 193.

[49] K.G. Chetyrkin, A.L. Kataev, and F.V. Tkachov, Phys. Lett. 85B (1979) 277; M. Dine and J. Sapirstein, Phys. Rev. Lett. 43 (1979) 668; W. Celmaster and R.J. Gonsalves, Phys. Rev. Lett. 44 (1980) 560. 
[50] L.R. Surguladze and M.A. Samuel, Phys. Rev. Lett. 66 (1991) 560; (E) 66 (1991) 2416; S.G. Gorishny, A.L. Kataev, and S.A. Larin, Phys. Lett. B259 (1991) 144.

[51] K.G. Chetyrkin, Phys. Lett. B307 (1993) 169.

[52] D.E. Soper and L.R. Surguladze, University of Oregon Report No. OITS 545, hepph/9406407 (June 1994).

[53] B.A. Kniehl, Phys. Lett. B237 (1990) 127.

[54] A.H. Hoang, M. Jeżabek, J.H. Kühn, and T. Teubner, Phys. Lett. B325 (1994) 495; (E) B327 (1994) 439; Karlsruhe University Report No. TTP94-11, hep-ph/9407338 (June 1994).

[55] A.L. Kataev and V.V. Starshenko, CERN Report No. CERN-TH.7198/94, hepph/9405294 (May 1994); CERN Report No. CERN-TH.7400/94, hep-ph/9408395 (August 1994), to appear in Proceedings of the QCD-94 Workshop, Montpellier, France, July 7-13, 1994, ed. by S. Narison, Nucl. Phys. B (Proc. Suppl.).

[56] A.L. Kataev, Phys. Lett. B287 (1992) 209.

[57] W.H. Furry, Phys. Rev. 51 (1937) 125.

[58] B.A. Kniehl and J.H. Kühn, Phys. Lett. B224 (1989) 229; Nucl. Phys. B329 (1990) 547.

[59] K.G. Chetyrkin and J.H. Kühn, Phys. Lett. B308 (1993) 127.

[60] K.G. Chetyrkin and A. Kwiatkowski, Phys. Lett. B319 (1993) 307.

[61] S.A. Larin, T. van Ritbergen, and J.A.M. Vermaseren, Phys. Lett. B320 (1994) 159; K.G. Chetyrkin and O.V. Tarasov, Phys. Lett. B327 (1994) 114.

[62] L.D. Landau, Docl. Akad. Nauk USSR 60 (1948) 207 [Phys. Abstr. A52 (1949) 125]; C.N. Yang, Phys. Rev. 77 (1950) 242.

[63] V.N. Baĭer, É.A. Kurayev, and V.S. Fadin, Yad. Fiz. 31 (1980) 700 [Sov. J. Nucl. Phys. 31 (1980) 364]; M.L. Laursen, K.O. Mikaelian, and M.A. Samuel, Phys. Rev. D23 (1981) 2795; M.L. Laursen and M.A. Samuel, Z. Phys. C14 (1982) 325.

[64] S.-C. Lee and W.-C. Su, Phys. Rev. D38 (1988) 414; J.J. van der Bij and E.W.N. Glover, Nucl. Phys. B313 (1989) 237; R. Höpker and J.J. van der Bij, Phys. Rev. D49 (1994) 3779.

[65] S.G. Gorishny, A.L. Kataev, and S.A. Larin, Nuovo Cim. 92A (1986) 117; L.R. Surguladze, INR Report No. P-0644 (1989) (unpublished); Yad. Fiz. 50 (1989) 604 [Sov. J. Nucl. Phys. 50 (1989) 372]; University of Oregon Report No. OITS 557 (1994) (in preparation). 
[66] K.G. Chetyrkin and J.H. Kühn, Phys. Lett. B248 (1990) 359.

[67] K.G. Chetyrkin, J.H. Kühn, and A. Kwiatkowski, Phys. Lett. B282 (1992) 221; K.G. Chetyrkin and A. Kwiatkowski, Phys. Lett. B305 (1993) 285.

[68] K.G. Chetyrkin and A. Kwiatkowski, Z. Phys. C59 (1993) 525; L.R. Surguladze, University of Oregon Report No. OITS 554 (1994) (in preparation); private communication.

[69] J. Gasser and H. Leutwyler, Phys. Rep. 87 (1982) 77; L.J. Reinders, H.R. Rubinstein, and S. Yazaki, Phys. Rep. 127 (1985) 1.

[70] S. Narison, Phys. Lett. B197 (1987) 405; ibid. B216 (1989) 191; CERN Report No. CERN-TH.7405/94, hep-ph/9408376 (August 1994); C.A. Dominguez and N. Paver, Phys. Lett. B293 (1992) 197; C.A. Dominguez, G.R. Gluckman, and N. Paver, Phys. Lett. B333 (1994) 184.

[71] C.A. Dominguez and E. de Rafael, Ann. Phys. (NY) 174 (1987) 372; C.A. Dominguez, C. van Gend, and N. Paver, Phys. Lett. B253 (1991) 241.

[72] S.G. Gorishny, A.L. Kataev, S.A. Larin, and L.R. Surguladze, Mod. Phys. Lett. A5 (1990) 2703; Phys. Rev. D43 (1991) 1633.

[73] E. Gross, B.A. Kniehl, and G. Wolf, Z. Phys. C63 (1994) 417.

[74] M.D. Hildreth, T.L. Barklow, and D.L. Burke, Phys. Rev. D49 (1994) 3441.

[75] L. Resnick, M.K. Sundaresan, and P.J.S. Watson, Phys. Rev. D8 (1973) 172.

[76] J. Fleischer and F. Jegerlehner, Phys. Rev. D23 (1981) 2001; D.Yu. Bardin, B.M. Vilenskiı̌, P.Kh. Khristov, Yad. Fiz. 53 (1991) 240 [Sov. J. Nucl. Phys. 53 (1991) 152]; A. Dabelstein and W. Hollik, Z. Phys. C53 (1992) 507.

[77] B.A. Kniehl, Nucl. Phys. B376 (1992) 3.

[78] M.S. Chanowitz, M.A. Furman, and I. Hinchliffe, Phys. Lett. 78B (1978) 285; Nucl. Phys. B153 (1979) 402; Z. Hioki, Phys. Lett. B224 (1989) 417.

[79] B.A. Kniehl, Phys. Rev. D50 (1994) 3314.

[80] A. Djouadi and P. Gambino, Report No. UdeM-GPP-TH-94-02 and NYU-TH-94/06/01, hep-ph/9406431 (June 1994).

[81] A. Kwiatkowski and M. Steinhauser, Phys. Lett. B338 (1994) 66.

[82] B.A. Kniehl and M. Spira, DESY Report No. 94-102 (October 1994). 
[83] J. Ellis, M.K. Gaillard, and D.V. Nanopoulos, Nucl. Phys. B 106 (1976) 292; A.I. Vaŭnshteŭn, M.B. Voloshin, V.I. Zakharov, and M.A. Shifman, Yad. Fiz. 30 (1979) 1368 [Sov. J. Nucl. Phys. 30 (1979) 711]; A.I. Vaŭnshtel̆n, V.I. Zakharov, and M.A. Shifman, Usp. Fiz. Nauk 131 (1980) 537 [Sov. Phys. Usp. 23 (1980) 429]; L.B. Okun, Leptons and Quarks (North-Holland, Amsterdam, 1982), p. 229; M.B. Voloshin, Yad. Fiz. 44 (1986) 738 [Sov. J. Nucl. Phys. 44 (1986) 478]; M.A. Shifman, Usp. Fiz. Nauk 157 (1989) 561 [Sov. Phys. Usp. 32 (1989) 289]; J.F. Gunion, H.E. Haber, G. Kane, and S. Dawson, The Higgs Hunter's Guide (Addison-Wesley, Redwood City, 1990), p. 40.

[84] E. Braaten and J.P. Leveille, Phys. Rev. D22 (1980) 715; N. Sakai, Phys. Rev. D22 (1980) 2220; T. Inami and T. Kubota, Nucl. Phys. B179 (1981) 171; M. Drees and K. Hikasa, Phys. Lett. B240 (1990) 455; (E) B262 (1990) 497.

[85] K.G. Chetyrkin, J.H. Kühn, and A. Kwiatkowski, Karlsruhe University Report No. TTP94-10, hep-ph/9407271 (July 1994), to appear in Proceedings of the 1994 Zeuthen Workshop on Elementary Particle Theory: Physics at LEP200 and Beyond, Teupitz, April 10-15, 1994, ed. by J. Blümlein and T. Riemann, Nucl. Phys. B (Proc. Suppl.).

[86] A.L. Kataev and V.T. Kim, Mod. Phys. Lett. A9 (1994) 1309.

[87] F. Wilczek, Phys. Rev. Lett. 39 (1977) 1304; J. Ellis, M.K. Gaillard, D.V. Nanopoulos, and C.T. Sachrajda, Phys. Lett. 83B (1979) 339; T.G. Rizzo, Phys. Rev. D22 (1980) 178; ibid. D22 (1980) 1824.

[88] T. Inami, T. Kubota, and Y. Okada, Z. Phys. C18 (1983) 69; A. Djouadi, M. Spira, and P.M. Zerwas, Phys. Lett. B264 (1991) 440.

[89] H. Zheng and D. Wu, Phys. Rev. D42 (1990) 3760; A. Djouadi, M. Spira, J.J. van der Bij, and P.M. Zerwas, Phys. Lett. B257 (1991) 187; S. Dawson and R.P. Kauffman, Phys. Rev. D47 (1993) 1264; A. Djouadi, M. Spira, and P.M. Zerwas, Phys. Lett. B311 (1993) 255; K. Melnikov and O. Yakovlev, Phys. Lett. B312 (1993) 179; M. Inoue, R. Najima, T. Oka, and J. Saito, Mod. Phys. Lett. A9 (1994) 1189; J. Fleischer and O.V. Tarasov, Bielefeld University Report No. BI-TP 93-78, hep-ph/9403230 (March 1994), Z. Phys. C (to appear).

[90] M. Spira, A. Djouadi, and P.M. Zerwas, Phys. Lett. B276 (1992) 350.

[91] B.A. Kniehl, DESY Report No. 94-036, hep-ph/9403386 (March 1994).

[92] A.I. Bochkarev and R.S. Willey, University of Pittsburgh Report No. PITT-TH-94-04, hep-ph/9407261 (June 1994). 